
REVISÃO SISTEMÁTICA

\title{
PRESENCE OF Rhodotorula IN HOSPITAL ENVIRONMENT: A SYSTEMATIC REVIEW
}

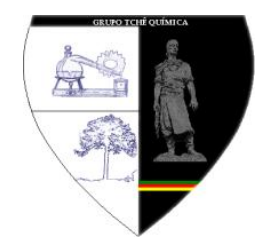

\author{
ANDRADE JÚNIOR, Francisco Patricio de ${ }^{1 *}$; CRUZ, José Henrique de Araújo'; OLIVEIRA \\ FILHO, Abrahão Alves de';
}

${ }^{1}$ Universidade Federal de Campina Grande, Centro de Educação e Saúde, Departamento de Farmácia, Olho D'água da Bica S/N, cep 58175-000, Cuité-PB, Brasil.

(fone: +55 084 8135-7242)

2Universidade Federal de Campina Grande, Centro de Saúde e Tecnologia Rural, Departamento de Odontologia, Avenida Universitária S/N, cep 58708-110, Patos-PB, Brasil.

(fone: +55 083 9625-0125)

3 Prof. Dr. da Universidade Federal de Campina Grande, Centro de Saúde e Tecnologia Rural, Departamento de Odontologia, Avenida Universitária S/N, cep 58708-110, Patos-PB, Brasil.

(fonte: +55 083 3511-3000)

\author{
${ }^{*}$ Autor correspondente \\ e-mail: juniorfarmacia.ufcg@outlook.com
}

Received 21 April 2018; received in revised form 10 June 2018; accepted 13 June 2018

\section{RESUMO}

Fungos do gênero Rhodotorula possuem a capacidade de causar o desenvolvimento de infecções, sobretudo, em pacientes imunodeprimidos podendo levá-los a óbito. O presente estudo teve como objetivo fazer uma revisão bibliográfica do tipo sistemática acerca da presença de Rhodotorula sp. em ambiente hospitalar, enfocando mãos de profissionais da saúde, superfícies de objetos presentes em hospitais e 0 ar. $O$ levantamento bibliográfico ocorreu nas bases de dados Scielo, Lilacs, PubMed, Science Direct e Periódicos Capes, de artigos publicados entre os anos 2000 à 2017. Na revisão de literatura houve a recuperação de 3164 publicações, das quais 56 tiveram seus textos avaliados por completo e somente 7 foram selecionadas para compor os resultados. A prevalência de Rhodotorula sp. nos diferentes estudos variou entre $3,3 \%$ à $23,7 \%$, onde ao total observou-se 1170 amostras contaminadas por fungos, das quais o gênero Rhodotorula foi encontrado em 71 amostras $(6,1 \%)$, em que $13 \%(n=9)$ foram detectados em superfícies de objetos e equipamentos, $14 \%(n=10)$ foram encontrados em mãos de profissionais da saúde e $73 \%(n=52)$ no ar. Deste modo, é necessário que medidas mais rígidas de limpeza sejam adotadas pelos hospitais a fim de diminuir a presença desses microrganismos e impedir o desenvolvimento de infecções.

Palavras-chave: Micologia, Leveduras, Gênero Rhodotorula, Epidemiologia, Saúde Pública.

\section{ABSTRACT}

Fungi of the genus Rhodotorula have the capacity to cause the development of infections, especially in immunosuppressed patients and can lead to death. This study aimed to make a systematic review of the Rhodotorula sp. in a hospital environment, focusing hands of health professionals, surfaces of objects present in hospitals and the air. The bibliographic survey was carried out in the databases Scielo, Lilacs, PubMed, Science Direct and Periodical Capes, of articles published between the years 2000 to 2017. In the literature review, 3164 publications were retrieved, of which 56 had their texts fully evaluated and only 7 were selected to compose the results. The prevalence of Rhodotorula sp. in the different studies ranged from $3.3 \%$ to $23.7 \%$, where 1170 samples were contaminated by fungi, of which the genus Rhodotorula was found in 71 samples $(6.1 \%)$, where $13 \%=9)$ were detected on surfaces of objects and equipment, $14 \%(n=10)$ were found in the hands of health professionals and $73 \%(n=52)$ in the air. In this way, it is necessary that more strict measures of cleaning are adopted by the hospitals in order to diminish the presence of these microorganisms and to prevent the

PERIÓDICO TCHÊ QUÍMICA • www.periodico.tchequimica.com • Vol. 16 N. 31.

• ISSN 1806-0374 (impresso) • ISSN 1806-9827 (CD-ROM) • ISSN 2179-0302 (meio eletrônico)

(C) 2019. Porto Alegre, RS. Brasil 
development of infections.

Keywords: Mycology, Yeasts, Rhodotorula genus, Epidemiology, Public Health.

\section{INTRODUÇÃO}

Os fungos são microrganismos ubiquitários e eucariotos que contêm um núcleo bem definido, mitocôndria, complexo de Golgi, retículo endoplasmático e podem existir em forma unicelular (leveduras) que se replica assexuadamente ou na forma pluricelular (fungos filamentosos) podendo se replicar sexuadamente e assexuadamente (MURRAY; ROSENTHAL; PFALLER, 2014).

Estes eucariontes vêm ganhando importância no decorrer dos últimos anos devido aos fenômenos de resistência, a sua capacidade de produzir micotoxinas que contribuem para a instabilidade da saúde huamana e principalmente, devido a infecções nosocomais, que ocupam o terceiro ou quarto lugar como agentes etiológicos responsáveis (PEMÁN; ZARAGOZA; SALAVERT; 2013; ANDRADE JÚNIOR et al., 2018).

Esta situação representa um risco à saúde pública, sendo necessário que haja a investigação de quais espécies ou gêneros fúngicos possam ser encontrados em ambientes internos de áreas críticas hospitalares, em mãos de profissionais da saúde e em superfícies de objetos presentes em hospitais (RUIZ; PEREIRA, 2016).

Dentre os gêneros fúngicos de interesse hospitalar, é possível destacar o gênero Rhodotorula que se caracteriza por ser uma levedura comensal da microbiota normal de seres humanos (pele, unhas e mucosas). Compostos por células de brotamento multilateral estes fungos são capazes de produzir pigmentos carotenoides, dando as suas colônias a coloração que varia entre rosa e vermelho possuindo como principais espécies $R$. glutinis, $R$. mucilaginosa e $R$. minuta (Figura 1) (MURRAY; ROSENTHAL; PFALLER, 2014; ALMEIDA, 2005).

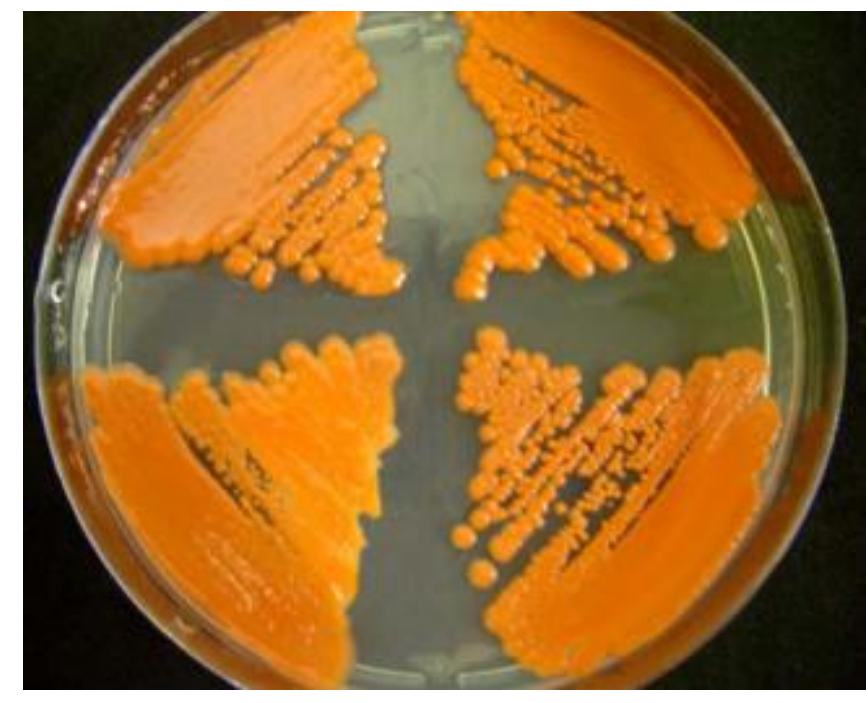

Figura 1. Características macroscópicas de Rhodotorula sp.

Fonte: ALMEIDA, 2005

Este gênero fúngico é capaz de causar infecções oportunistas, tratando-se, portanto, de infecções que se desenvolvem em pacientes que possuem a função fagocitária deprimida, seja devido disfunções metabólicas, doenças crônicas, terapia imunodepressora ou esteroide (SILVA et al., 2014), podendo ocasionar em graves enfermidades, como meningite e endocardite (LOSS, 2011).

Deste modo, levando em consideração a importância que fungos do gênero Rhodotorula podem apresentar quanto agentes etiológicos em pacientes imunocomprometidos, 0 presente estudo teve como objetivo fazer uma revisão bibliográfica do tipo sistemática acerca da presença de Rhodotorula sp. em ambiente hospitalar seja por meio de mãos de profissionais da saúde, superfícies de objetos presentes em hospitais ou advindos do ar.

\section{MATERIAL E MÉTODOS}

\subsection{Delineamento do Estudo}

O presente estudo trata-se de uma revisão bibliográfica do tipo sistemática, uma vez que, houve a utilização de dados advindos da literatura utilizando rigor metodológico de modo sistemático permitindo a reprodutibilidade de 

2007).

\subsection{Critérios de Inclusão e Exclusão}

Foram incluídos neste estudo artigos que, em seu conteúdo, trouxessem informações quantitativas acerca da presença de Rhodotorula sp. em mãos de profissionais de saúde, superfície de objetos e no próprio ambiente hospitalar (ar), de artigos publicados entre os anos 2000 à 2017 que se apresentassem em língua inglesa, portuguesa e espanhola. Trabalhos em que não foram constatados a presença de Rhodotorula sp. ou que foram publicados fora do tempo cronológico delimitado, foram excluídos do estudo.

\subsection{Fontes de Informação}

Os artigos foram recuperados a partir das bases de dados: Lilacs (Centro América Latina e Caribe em Ciências da Saúde), Scielo (Scientific Eletronic Library Online), Science Direct, PubMed e Periódicos Capes. O levatamento de dados ocorreu entre novembro de 2017 à janeiro de 2018.

\subsection{Estratégia de Busca}

A estratégia de busca utilizada para pesquisa nas bases de dados se deu pela utilização dos seguintes descritores e palavraschave: ("Rhodotorula" AND "Hospital") OR ("Rhodotorula" AND "Nosocomial") OR ("Rhodotorula" AND "hospital environment").

\subsection{Extração dos Dados}

Foram extraídas as seguintes variáveis: autor(es), ano de publicação da pesquisa, origem da amostra coletada (superfície de objetos e equipamentos presentes no hospital, mãos de profissionais da saúde ou o próprio ambiente), quantidade de amostras positivas para fungos $e$ quantidade de amostras especificamente positivas para Rhodotorula.

\section{RESULTADOS E DISCUSSÃO}

$\mathrm{Na}$ revisão de literatura houve a recuperação de 3164 publicações, das quais 56 tiveram seus textos avaliados por completo. Por fim, somente 7 artigos foram incluídos para compor os resultados (Figura 1).

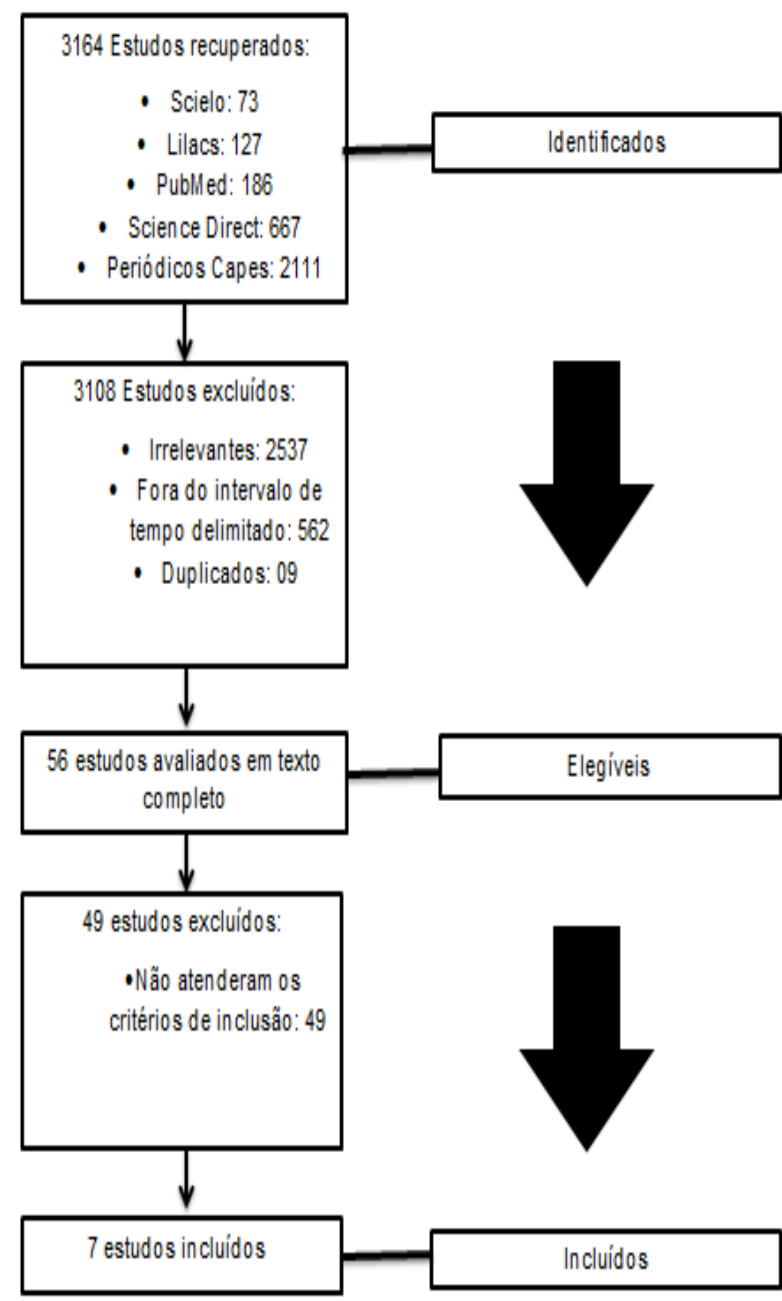

Figura 2. Processo de busca, seleção e inclusão de artigos.

A presença de Rhodotorula em hospitais pode ser constatada em estudos publicados entre os anos 2000 à 2015, como pode ser observado na tabela 1 .

A prevalência de Rhodotorula sp. nos diferentes estudos variou entre $3,3 \%$ à $23,7 \%$, onde ao total observou-se 1170 amostras contaminadas por fungos, das quais o gênero Rhodotorula foi encontrado em 71 amostras $(6,1 \%)$.

A constatação de Rhodotorula em 6,1\% das amostras é um dado importante, uma vez que, este gênero fúngico tem a capacidade de agir como oportunista em pacientes imunocomprometidos (MURRAY; ROSENTHAL; PFALLER, 2014; MARTÍNEZ et al., 2013).

Assim, é preciso que os hospitais utilizem medidas para evitar a presença não somente de fungos patogênicos, mas também de oportunistas, para isso é necessário que se conheça quais são as fontes de contaminação ou 
onde estes fungos possam ser encontrados dentro do ambiente hospitalar.

Abaixo é possível constatar o percentual de Rhodotorula encontrada no ar, em mãos e objetivos/equipamentos presentes em ambiente hospitalar (Figura 3).

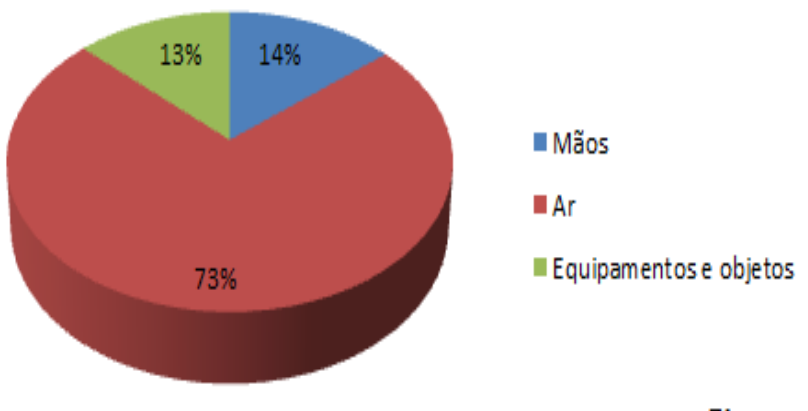

Figura 3. Detecção de Rhodotorula no ar de ambientes hospitalares, mãos de profissionais da saúde e em objetos e equipamentos de uso hospitalar.

Nos estudos de García e Valles (2014), Carrilo-Dover et al., (2009) e Passos et al., (2000) é possível observar a presença de Rhodotorula sp. em mãos de profissionais da saúde, equivalente a $14 \%$ das amostras contaminadas.

As mãos são as principais ferramentas de trabalho utilizadas por profissionais da saúde, contudo constituem a principal via de transmissão de microrganismos ao paciente, uma vez que, podem ser verdadeiros reservatórios de patógenos (SANTOS; GONÇALVES, 2009). Mesmo tratando-se de um fungo não patogênico, a presença de espécies de Rhodotorula sp. nas mãos, pode ser considerada como um forte indicador de possível colonização de outras leveduras, já que estes órgãos estão associados a grandes números de infecções hospitalares (GARCÍA; VALLES, 2014; XISTO et al., 2000).

Além disso, as mãos devem ser bem higienizadas, pois podem desempenhar o papel de fonte de transmissão de microrganismos a objetos utilizados em procedimentos invasivos e/ou promover a transmissão de infecções veiculadas pelo contato direto ao paciente (BRASIL, 2007). A presença de microrganismos em mãos, pode também está relacionada ao tipo de antisséptico utilizado para higienização destes órgãos. Em um estudo realizado em profissionais de saúde, observou-se que higienização com solução alcoólica é mais eficaz quando comparada a técnica de escovação com digliconato de clorexidina 2\% (MENEZES et al., 2016), enquanto que em um estudo realizado com diversos antissépticos, observou-se que a assepsia das mãos com álcool é mais eficaz do que a lavagem prévia utilizando água e sabão. Contudo o uso do álcool como desinfetante ainda é pouco aceito no Brasil (VOLKART et al., 2017), podendo porém ser mais uma medida implementada para assegurar a limpeza das mãos antes que os profissionais de saúde entrem em contato com o paciente.

Nos estudos de García e Valles (2014) e Gonçalves et al., (2015) foi observada a presença de Rhodotorula em superfícies de objetos e equipamentos, correspondendo a $13 \%$ das amostras contaminadas.

Outros estudos também evidenciam a presença de fungos em artigos médicohospitalares, objetos e equipamentos. Em um hospital de Araraquara-SP, 91 amostras foram colhidas do mobiliário em que $44 \%$ foram positivas para leveduras, tendo a Candida como gênero predominante $(70 \%$ ) (MARTINS-DINIZ et al., 2005), enquanto que no município de Francisco Beltrão-PR, ao analisar maçanetas e cabeceiras de cama de uma unidade hospitalar detectou-se mais abundantemente a presença de colônias fúngicas do gênero Cladosporium spp, Fusarium spp e Penicillium spp. (FLORES; ONOFRE, 2010). Em um hospital no interior de São Paulo, realizaram uma investigação microbiológica em leitos hospitalares, onde observou-se que de 520 placas, o crescimento de fungos foi evidenciado em $42,1 \%$ destas amostras, entretanto as espécies e/ou gêneros envolvidos não foram identificados (ANDRADE; ANGERAMI; PADOVANI, 2000). Em Pouso Alegre-MG, verificou-se em 30 amostras a presença de 160 colônicas fúngicas, sendo que destas $40 \%$ correspondiam a fungos do gênero Penicillium (MELO et al., 2009).

Este dado é alarmante, uma vez que, a utilização de sondas, cateteres ou outros materiais médico-hospitalares invasivos podem contribuir para a contaminação de pacientes imunocomprometidos (MARTÍNEZ et al., 2013), uma vez que, a inserção destes instrumentos destroem a epiderme permitindo a penetração e o possível desenvolvimento de microrganismos, causando assim, o desenvolvimento de infecções (ANDRADE JÚNIOR et al., 2017). Ademais, estudos demonstram que cepas de Rhodotorula 
sp. em equipamentos médicos e mãos de profissionais da saúde têm se mostrado resistentes aos triazólicos e equinocandinas (GARCÍA; VALLES, 2014; LUNARDI et al., 2006) dificultando cada vez mais o tratamento daqueles que forem acometidos, trazendo, consequentemente, mais gastos aos hospitais que terão de manter o paciente hospitalizado por mais tempo e utilizando antifúngicos cada vez mais potentes e dispendiosos.

A correta higienização de objetos $e$ equipamentos hospitalares implicam na eleição certa de um saneante que seja eficiente contra a maioria dos microrganismos de interesse médico.

O hipoclorito de sódio e sabão neutro são amplamente utilizados para a limpeza de superfícies e alguns equipamentos, entretanto é evidenciado que a forma de diluição desses saneantes podem contribuir para imperfeita desinfecção, enquanto que a utilização do óxido de etileno (ETO) tem se apresentado como um interessante agente, uma vez que possui atividade esterilizante. Quando o ETO entra em contato com equipamentos ou materiais médicohospitalares, este se liga irreversivelmente as bases nitrogenadas dos microrganismos que neles estejam presentes, impedindo portanto a duplicação do material genético, impossibilitando assim o desenvolvimento de outras células. Outras opções que podem ser considerados para limpeza desses fômites e que são amplamente discutidas na literatura é o glutaraldeído, considerado um desinfetante de alto nível por matar uma ampla gama de microrganismos, entretanto devido falhas durante o reprocessamento de artigos médico-hospitalares, que não atingiam o tempo mínimo de imersão, houve o aparecimento de cepas tolerantes, sendo portanto proibida a sua utilização para alguns artigos invasivos, enquanto que no caso do ácido peracético é utilizado para a esterilização e desinfecção de alto nível impedindo a sobrevivência e reprodução de microrganismos em artigos médico-hospitalares de uso crítico e semicrítico (VENCESLAU; MARTINS; OLIVEIRA, 2012; SILVA et al., 2017; BARBOSA; SARTORI, 2017).

A presença de espécies do gênero Rhodotorula no ar foi responsável por $73 \%$ das amostras contaminadas, o que pode ser atribuído a instalação de ambientes com climas artificiais dando a esses microrganismos um sítio adequado a proliferação (SANTANA; FORTUNA, 2012).
Outros hospitais também evidenciaram a presença de fungos no ar. Em um hospital localizado na cidade de Ariquemes- $\mathrm{RO}$, foi visto que de 50 amostras $20 \%$ destas estavam contaminadas Fusarium spp., 14\% por Curvularia sp e $12 \%$ com Cladosporium sp. (PEREIRA et al., 2014). Enquanto que no município de Rio Grande-RS, foram analisadas 104 amostras de ar, sendo que os fungos mais prevalentes encontrados foram Cladosporium spp (75\%), Aspergillus spp $(71,15 \%)$ e Alternaria spp (53,85\%) (LOBATO; VARGAS; SILVEIRA, 2009).

Outros fatores causais relacionados a presença de fungos no ar são: a falta de controle da ventilação, a falta de limpeza e manutenção de ar condicionado, assim como, a entrada de pessoas, materiais, poeira, janelas abertas e a forma de limpeza ineficaz (PEREIRA et al., 2014; CENTENO; MACHADO, 2004).

Logo, esse excesso de contaminação pelo ar, pode propiciar a instalação de outros fungos anemófilos, a exemplo de Candida, Aspergillus e Penicillium (GONÇALVES et al., 2017) podendo ocasionar, inclusive, em complicações ainda maiores para os pacientes imunocomprometidos e possivelmente para pacientes imunocompetentes uma vez que tratam-se de fungos altamente patogênicos.

Mesmo dando-se menor importância a Rhodotorula sp., por ser considerado um fungo oportunista, sabe-se que a infecção causada por este gênero tem sido associada a altas taxas de mortalidade, correspondendo a uma cifra superior a 15\% (ROSA, 2013). Em um estudo de revisão, observou-se 128 casos de infecções causadas por Rhodotorula, onde $87 \%$ dos casos se deu em pacientes imunocomprometidos; a fugemia esteve presente em $79 \%$ dos casos, seguidos de infecções oculares e peritonites, com $7 \%$ e $5 \%$ dos casos respectivamente, enquanto que a mortalidade esteve presente em $12,6 \%$ dos casos (TUON; COSTA, 2008).

Contudo, uma limpeza correta no ambiente hospitalar, é considerada a melhor alternativa para o controle da contaminação fúngica, uma vez que, quanto maior a limpeza, maior será a segurança do ambiente hospitalar (HONORATO, 2009).

É importante ressaltar que este é o primeiro estudo de revisão que trata sobre a contaminação de Rhodotorula em ambiente hospitalar, podendo portanto servir como foco centralizador de outras pesquisas que envolvam 
esta temática e também como um alerta para que os gestores de saúde possam criar políticas públicas que permitam contribuir para a diminuição da contaminação fúngica em ambiente hospitalar, seja através da capacitação constante dos profissionais, de medidas mais restritivas acerca da livre circulação de pessoas em ambientes hospitalares, utilização de filtros HEPA e a descontaminação correta de objetos

\section{CONCLUSÃO}

A contaminação por fungos do gênero Rhodotorula foi considerada alta $(6,1 \%)$ em amostras advindas de mãos de profissionais da saúde, superfícies de objetos hospitalares e o próprio ambiente hospitalar tratando-se de um dado preocupante. Entretanto, algumas medidas preventivas podem ser tomadas para reduzir a presença de fungos em hospitais, como a lavagem correta das mãos, o controle da entrada de pessoas e do ar e a higienização correta de superfícies.

Mais estudos precisam ser realizados a nível nacional e internacional, para que se conheça o perfil microbiológico dos mais diversos hospitais permitindo, assim, a construção de medidas efetivas que, por sua vez, contribuirão para a quebra da cadeia de contaminação.

\section{REFERÊNCIAS}

1. Acta paulista de enfermagem. Acta Paul Enfer., 2007, 20.

2. Almeida, G. M. D. Rhodotorula spp. isoladas de hemocultura no Hospital das clínicas da Faculdade de Medicina da Universidade de São Paulo: características clínicas e microbiológicas. 2005. 139 f. Tese (Doutorado em Ciências) - Universidade de São Paulo, São Paulo, 2005.

3. Andrade Júnior, F. P., Alves, T. W. B., Romano, T. K. F., Medeiros, F. D. Periódico Tchê Química, 2018, 15, 321.

4. Andrade Júnior, F. P., Alves, T. W. B., Lima, B. T. M., Dantas, B. B. Atividade antiestafilocócica da Anacardium occidentale: uma revisão. In: One, G. M. C. (Org.). Campina Grande: Instituto Bioeducação, 2017, p.299-314.

5. Andrade, D., Angerami, E. L. S.,
Padovani, C. R. Revista de Saúde Pública, 2000, 34, 169.

6. Barbosa, L. S., Sartori, M. R. K. Cadernos da Escola de Saúde, 2017, 1.

7. Brasil. Agência Nacional de Vigilância Sanitária. Higienização das mãos em serviços de saúde. Brasília: Anvisa, 2007. $52 \mathrm{p}$.

8. Carrillo-Dover, P., Álvarez-Veja, C., Salas-Campos, I., Mora-Brenes, N.. Acta Médica Costarricense, 2009, 51, 171.

9. Centeno, S.; Machado, S. Investigación Clínica, 2004, 4, 144.

10. Cordeiro, R. A., Brilhante, R. S. N., Pantoja, L. D. M., Moreira Filho, R. E., Vieira, P. R. N., Rocha, M. F. G., Monteiro, A. J., Sidrim, J. J. C. Braz J Infect Dis., 2010, 14, 34.

11. Flores, L. H., Onofre, S. B. Revista de Saúde e Biologia, 2010, 5, 26.

12. García, Y. S., Valles, R. H. Revista de la Sociedad Venezolana de Microbiología, 2014, 34, 32.

13. Gonçalves, C. L., Mota, F. V., Ferreira, G. F., Mendes, J. F., Pereira, E. C., Freitas, C. H., Vieira, J. N., Villarreal, J. P., Nascente, P. S. Brazilian Journal of Biology, 2017.

14. Gonçalves, C. L., Mota, F. V., Mendes, J. F., Ferreira, G. F., Vieira, N. J., Pereira, E., Nascente, P. S. Revista de Epidemiologia e Controle de Infecção, 2015, 5, 112.

15. Honorato, G. M. Revista Eletrônica de Biologia, 2009, 2, 31.

16. Lobato, R. C.; Vargas, V. S.; Silveira, E. S. Revista da Faculdade de Ciências Médicas, 2009, 11, 28.

17. Loss, S. H., Antonio, A. C. P., Roerhrig, C., Castro, P. S., Maccari, J. G. Rev Bras Ter Intensiva, 2011, 23, 509.

18. Lunardi, L. W., Aquino, V. R., Zimerman, R. A., Goldani, L. Z. Clin Infect Dis, 2006, 43, 63.

19. Martínez, I. R., Morales, L. P., García, M. M., Castillo, J. E. B. MediSur, 2013, 11, 545.

20. Melo, L. L. S., Lima, A. M. C., 
Damasceno, C. A. V., Vieira, A. L. P. Revista Paulista Pediátrica, 2009, 27, 308.

21. Menezes, R. M., Cardoso, V., Hoehr, C. F.; Bulle, D.; Burgos, M. S.; Benitez, L. B.; Renner, J. D. P. Revista de Epidemiologia e Controle de Infecção, 2016, 6.

22. Murray, P.R.; Rosenthal, K.S.; Pfaller, M.A. Microbiologia Médica. 7. ed. Rio de Janeiro: Elsevier, 2014.

23. Pantoja, L. D. M., Couto, M. S., Leitão Júnior, N. P., Sousa, B. L., Mourão, C. I., Paixão, G. C. Rev Bras Promoç Saúde, 2012, 25, 196.

24. Passos, X. S., Souza, L. H., Costa, M., Costa, C. R., Fernandes, O. F. L., Silva, M. R. R. Revista de Patologia Tropical, 2000, 29, 203.

25. Pemán, J., Zaragoza, R., Salaver, M. Rev Esp Quimioter., 2013, 26, 311.

26. Pereira, J. G., Zan, R. A., Jardin, C. F., Meneguetti, D. U. O. Revista de Epidemiologia e Controle de Infecção, 2014, 4, 22.

27. Pereira, J. G., Zan, R. A., Jardin, C. F., Meneguetti, D. U. O. Revista de Epidemiologia e Controle de Infecção.

28. ROSA, M. A. L. Monitorização da presença de fungos com interesse clínico em ambiente hospitalar: zonas de banho como potenciais focos de contaminação fúngica. 2013. 99 f. Dissertação (Mestrado em Engenharia do Ambiente) Universidade dos Açores, Angra do Heroísmo, 2013.

29. Ruiz, L. S., Pereira, V. B. R. Bol Inst Adolfo Lutz, 2016, 26, 3.

30. Santana, W. O., Fortuna, J. L. Revista Biociências, 2012, 18, 64.

31. Santos, F. M., Gonçalves, V. M. S. Revista Enfermagem Integrada, 2009, 2, 163.

32. Silva, A. K. F., Lisboa, J. E. S., Barbosa, M. P. C. S., Lima, A. F. Cadernos de Graduação, 2014, 2, 47.

33. Silva, R. M., Sandri, A. P. G., Nakano, V., Nishiyama, S. A. B. REVISTA UNINGÁ, 2017, 4.

34. Tuon, F. F.; Costa, S. F. Revista
Iberoamericana de Micologia, 2008, 25, 140.

35. Venceslau, E. M., Martins, R. P. P, Oliveira, I. D. RBAC, 2012, 44, 30.

36. Volkart, P. A., Spagiari, M. S., Bizani, D. Arq. Cienc. Saúde UNIPAR, 2017, 20, 32. 
Tabela 1. Presença de Rhodotorula em ambiente hospitalar de estudos publicados entre os anos 2000-2015.

\begin{tabular}{ccccc}
\hline Autor & Ano & $\begin{array}{c}\text { Origem da } \\
\text { amostra }\end{array}$ & $\begin{array}{c}\text { Amostras } \\
\text { contaminadas } \\
\text { por fungos }\end{array}$ & $\begin{array}{c}\text { Amostras } \\
\text { contaminas } \\
\text { por } \\
\text { Rhodotorula }\end{array}$ \\
\hline $\begin{array}{c}\text { García e } \\
\text { Valles }\end{array}$ & 2014 & $\begin{array}{c}\text { Mãos de } \\
\text { profissionais } \\
\text { da saúde, } \\
\text { equipamentos } \\
\text { e mobília }\end{array}$ & 38 & $4(11,5 \%)$ \\
$\begin{array}{c}\text { Centeno e } \\
\text { Machado }\end{array}$ & 2004 & Ar & 714 & $20(2,80 \%)$ \\
& \multicolumn{5}{c}{}
\end{tabular}

$\begin{array}{ccccc}\text { Cordeiro et al. } & 2010 & \text { Ar } & 80 & 19(23,7 \%) \\ \text { Passos et al. } & 2000 & \begin{array}{c}\text { Mãos de } \\ \text { profissionais } \\ \text { da saúde }\end{array} & 30 & 1(3,3 \%) \\ & \quad \begin{array}{c}\text { da } \\ \text { Pand }\end{array} & & \end{array}$

$\begin{array}{ccccc}\text { Pantoja et al. } & 2012 & \mathrm{Ar} & 155 & 13(8,4 \%) \\ \begin{array}{c}\text { Gonçalves et } \\ \text { al. }\end{array} & 2015 & \begin{array}{c}\text { Leitos e } \\ \text { Bancas }\end{array} & 29 & 6(20,7 \%)\end{array}$

$\begin{array}{ccccc}\begin{array}{c}\text { Carrilo-Dover } \\ \text { et al. }\end{array} & 2009 & \begin{array}{c}\text { Mãos de } \\ \text { profissionais } \\ \text { de saúde }\end{array} & 97 & 8(7,8 \%) \\ & & \end{array}$

Fonte: Autores, 2018.

PERIÓDICO TCHÊ QUÍMICA • www.periodico.tchequimica.com • Vol. 16 N. 31

• ISSN 1806-0374 (impresso) • ISSN 1806-9827 (CD-ROM) • ISSN 2179-0302 (meio eletrônico)

(C) 2019. Porto Alegre, RS. Brasil 